\title{
Developing a Feasible and Maintainable Ontology for Automatic Landscape Design
}

\author{
Pintescu Alina \\ Faculty of Engineering \\ Technical University of Cluj-Napoca \\ Cluj-Napoca, Romania \\ Matei Oliviu-Dorin \\ Faculty of Engineering \\ Technical University of Cluj-Napoca \\ Cluj-Napoca, Romania
}

\author{
Boancă Iuliana Păuniţa \\ Department of Land Measurements and Science \\ University of Agricultural Science and Veterinary Medicine \\ Cluj-Napoca, Romania \\ Honoriu Vălean \\ Automation Department \\ Technical University of Cluj-Napoca \\ Cluj-Napoca, Romania
}

\begin{abstract}
In general, landscape architecture includes analysis, planning, design, administration and management of natural and artificial. An important aspect is the formation of socalled sustainable landscapes that allow maximum use of the environment, natural resources and promote sustainable restoration of ecosystems. For such purposes, a designer needs a complete database with existing and suitable plants, but no designing tool has one. Therefore it is presented the structure and the development of on ontology suitable for storing and managing all information and knowledge about plants. The advantage is that the format of the ontology allows the storage of any plant species (e.g. live or fossil) and automated reasoning. Ontology is a formal conceptualization of a particular knowledge about the world, through the explicit representation of basic concepts, relations, and inference rules about themselves. Therefore the ontology may be used by a design tool for helping the designer and choosing the best options for a sustainable landscape.
\end{abstract}

Keywords-environment; landscapes; ontology; ontology-based simulation; sustainable landscapes

\section{INTRODUCTION}

In Romania studies on the landscape made up in the years 1990-1995 aimed especially theoretical approaches and descriptive works, the landscape is analysed through the interaction of natural components. The study of landscape was frequently included in physical-geographical studies. In Europe and the U.S. separation of theoretical and descriptive landscape analysis took place in 1960 when he moved to the quantitative analysis of specific aspects of the landscape. Thus, in 1980 the landscape starts to emerge Science, studying the organization of landscape, natural and human interrelations between components and their temporal dynamics.

There are several application used in landscape design, applications which make use databases and ontology with certain plants. However, these databases or ontology lack many attributes of the plants and are useless for non-specialists. The designers must make use of their experience and background knowledge to use the existing information. Therefore, the aim of the next article is the design and implementation of ontology about plants used in sustainable landscape design, along with their properties and relationships.

This paper presents the structure and the development of ontology suitable for storing and managing all information and knowledge about plants. The rest of the paper is organized as follows. Section 2 presents the related work in the field of sustainable landscape design. Section 3 discusses the structure and the development of on ontology suitable for storing and managing all information and knowledge about plants. In Section 4, the knowledge modelling environment in this study is presented. Section 5 contains the proccess to obtained the ontology. Section 6 discusses the results presented in section 5. Finally, conclusion and future work is presented in Section 7.

\section{RELATED WORK}

There are different viewpoints regarding sustainable landscape design and types of ontology. In [1] Selman claimed the fact that landscape has become a major issue in spatial policy both as a sector in its own right, important to outdoor amenity and the leisure economy, and, increasingly, as a basis for framing and managing wider socio environmental systems. This trend reflects two broad schools in sustainable landscape development - one focused on the design and protection of scenic assets and the other emphasizing dynamic multifunctional links between ecosystem services and human well-being.

The social dimension of sustainability has been growing in importance as a criterion for evaluating the viability of projects in the construction sector. Reference [2] presents an ontology that can be employed to provide a systematic articulation to the issues that impinge on the social dimension of sustainability appraisals. The development of the social ontology was a consequence of a research project that explored the tools, metrics and models employed in the evaluation of sustainability within the urban environment.

Creating sustainable landscapes is very important especially in the context of environmental conversion of closed mines ([3]) and landslides ([4]); in the context of the accentuated urbanization and of the negative implication on 
environment. There are even studies on the fossil flora by Macovei and Givulescu ([5]). Reference [6] tackles algorithms applied in Environmental sciences. Sustainability has been defined by Park ([7]), in part, as the ecological balance that allows us to meet our needs without compromising the ability of future generations to meet theirs.

Sustainable landscape planning is based on a variety of techniques and systems designed to manage environmental problems caused by different factors (increased urbanization, climate change, greenhouse effect). The systems or techniques of sustainable landscape planning is not addressed and not treats only one environmental problem. These systems are designed, most often to alleviate or treat several negative factors that are co-dependent (urbanization - air pollution noise pollution - greenhouse effect - urban high temperatures the increase of the impervious surfaces, the reduction of soil permeability - the increase of the volume and speed of the pluvial runoff runaway water - the pollution of the hydrographical network) ([8], [9]).

Even if the conventional landscapes have also an ecological function, generally focuses on the aesthetic function. Stoecklein said that a good landscape architect should have an extensive knowledge of plant, soils, and sites and eye for design, in order to make the right plant selections and put them artistically into their proper locations ([10]). Currently, the sustainable landscapes and the landscape architecture bring forward the ecological function ([11], [12], [13], [14], [15], [16]). Not only selection and artistic arrangement of the plant counts but also the environmental benefits and their functions according to the issues addressed.

Levinthal has considered the problem of landscape design like the tuning of fitness landscapes on which actors adapt ([17]). He examined how alternative organizational designs influence actors' fitness landscapes and, in turn, the behavior that these alternative designs engender. Reducing interdependencies leads to robust designs that result in relatively stable and predictable behaviors. Reference [18] provided an overview of established and emerging forces that influence landscape design. Management, planning, and design as appreciative systems and regenerative processes require the landscape be addressed as system, and the designer be actively engaged in integrative systems thinking. In "Ecological landscape design and planning" the authors contributed to holistic landscape research, creative landscape design and sustainable landscape planning ([19]).

The reference [20] illustrated that environmental quality is one of the factors that has a direct effect on the health and wellbeing of people in urban areas. They suggested that the urban environment is characterized by altered climate and water relations, damaged soils, man-made substrates, a specialized flora of native and non-native species, and a strong cultural context.

According to [21] sustainable design has a lot to do with society, economy and environment's principles. Social aspects of sustainability, is in need of community participation. Participation in the design process especially in landscape architecture and design is one of the most important factors which are emphasized in recent years and new theories.
Bata delimited the borders of areas, units or entities like universal problem in the spatial and landscape sciences, particularly when addressing natural systems or socioecological systems ([22]). The authors of [23] concluded that the best way to keep pace with the latest thinking in environmental planning and landscape design are: the fundamentals of landscape and environmental planning, nature as the all-embracing framework, topography and earth modeling, treatment of wetlands, waterways, and water bodies. The latest researches in the field of the sustainable landscape were focused also on spatial optimization model for landscape planning and renewable energy ([24], [25]).

Reference [26] described the creation of knowledge sharing system for sustainability science through the application of semantic data modeling. An ontology grounded in description logics was developed based on the ISO 15926 data model to describe three types of sustainability science conceptualizations: situational knowledge, analytic methods, and scenario frameworks. Semantic statements were then created using the ontology to describe expert knowledge expressed in research proposals and papers related to sustainability science and in scenarios for achieving sustainable societies.

An important function in the development of urban areas has their ecological assessment and the methods used ([27], [28]). There are various methods for environmental assessment. For the ecological (sustainable) landscape design are available various solutions of numerical modeling and selection of vegetation - but these solutions are not complete. The success of these landscapes is ensured by the high degree of interdisciplinary involved by design and implementation. Initial studies, numerical modeling and planning are important elements for achieving a viable sustainable landscape.

During the development of sustainable landscaping techniques appeared important tools for assessment, modeling, design and implementation. The inconvenience refers to the restriction of the use of these tools ([29]). These restrictions arise because the sustainable landscapes and the related techniques address local and regional conditions and depend on the existing factors in a particular area.

So, the modeling tools, design and selection of vegetation are often developed according to the existing conditions in a particular region of the world or a country, and cannot be applied only under similar conditions to other regions. The vegetation has the one of the most important roles in the proper functioning of the sustainable landscapes. In order to achieve the specific objectives, of a particular sustainable landscaping technique, vegetation should be chosen to optimize, support and comply with its functions. An advanced and complex ontology that include plants with various characteristics enable accurate selections based on the requirements of the techniques that are to be implemented, on local conditions, and on the functions that are intended to be met by the proposed landscape.

\section{STRUCTURE OF THE ONTOLOGY}

Ontology is formal conceptualization of a particular knowledge about the world, through the explicit representation 
of basic concepts, relations, and inference rules about themselves ([30], [31]). Domain ontology can be used to provide knowledge support in underlying cognitive processes and inter-relations.

Some reasons why someone would develop an ontology are described by [32]: to share common understanding of the structure of information among people or software agents, to enable reuse of domain knowledge, to make domain assumptions explicit, to separate domain knowledge from the operational knowledge, to analyze domain knowledge. Developing ontology is asking to define a set of data and their structure for other programs to use. Problem-solving methods, domain-independent applications, and software agents use ontology and knowledge bases built from ontology as data.

For example, according to [33] the necessity of creating a legal ontology specific to the Romanian juridical context resides in the fact that, although different state institutions hold various databases storing documents, there is a lack of centralization of these data.

The ontology for sustainable landscape design was developed. This ontology can be used as a basis for some applications in a suite of landscape-managing tools: the application could create the possible garden architecture suggestions for the each potential client. With such ontology as the one proposed in this article, software algorithms may be used to extract and match the suitable plants, such as evolutionary ontology ([34]).

Different definition of ontology provides various facilities. The latest development in defining standard languages OWL ontology is produced by World Wide Web Consortium (W3C). Like RDF, OWL makes it possible to describe concepts but in addition, provides many other facilities. It has a richer set of operators (for example and, or, negation). It is based on a different logical model that allows concepts to be simultaneously defined and described. Complex concepts can be defined based on simple concepts. The logical model allows use reasoners to check whether all the definitions and declarations are mutually consistent and can also acknowledges that fit concepts and under the definition. The function of reasoners helps to maintain a proper hierarchy. This hierarchy is useful when working with classes that have more than one parent. Unlike a mere database, such as many existing ones, an ontology is capable of describing the relationships between entities, e.g. what plants match what conditions, what plants need to be planted around what plants etc. Moreover, ontology is a good platform for reasoning and inference, very well described by [35]. This means that the user has all the tools to query the ontology and find out any existing characteristic about the approached domain.

\section{KNOWLEDGE MODELLING ENVIRONMENT}

Reference [36] presented methodologies for building ontology from scratch. Reference [37] and [38] proposed the solution for ontology based approaches. Typical ontology enabled tasks include profile matching, gap analysis and the selection of appropriate learning opportunities.

Ontology is a semantically enriched data model that represents a set of concepts within a domain and the relationships between those concepts ([39]) OWL ontology has similar components to Protégé frame based ontology. However, the terminology used to describe is different from that used by Protégé. OWL ontology is constructed of Individuals, Properties and classes that have correspondent in Protégé: Instance, Slots and Classes. Individuals represent objects in the domain that was interesting. An important difference between Protégé and OWL is that OWL does not use Unified Name Assumption (UNA), means that two distinct name can be the same individual.

For example, "Queen Elizabeth Rose", "Queen Elizabeth" or "Elizabeth Rose" assigned the same individual. OWL have explicitly stated that individuals are equally distinctive each other. Individuals are also known as instances and may be referred to as "instances of classes". The properties are binary relations between individuals like individual property of the two links each other. For example, property can link individual isToxic for Bluebells (in Romanian Viorele) and for individual MayLily (in Romanian Lăcrimioare), or property Content can bind Plants for Flowers. Properties can be reversed. Such as the inverse of isToxic is notToxic. The properties can be limited to have a single value. Also they can be transitive or symmetric. Properties are equivalent slots in Protégé. They are also known as logical description of roles and relationships in UML and other object-oriented notations.

\section{DEVELOPING THE ONTOLOGY}

The Artificial-Intelligence literature contains many definitions of ontology, many of these contradict one another ([32]): ontology is a formal explicit description of concepts in a domain of discourse (classes - sometimes called concepts), properties of each concept describing various features and attributes of the concept (slots - sometimes called roles or properties), and restrictions on slots (facets - sometimes called role restrictions).

There are three basic rules for building ontology. These rules help to make decisions in a variety of situations. There is no one correct way to model a domain - there are always alternatives ([32]). The best solution usually depends on the application that builds the ontology and the extent who can expect. Building ontology is an iterative process.

Ontology together with a set of individual instances for classes constitutes a knowledge base. In reality, there is a fine line where the ontology ends and the knowledge base begins. Classes describe concepts in the domain. OWL classes are interpreted as sets which contain individuals. They are those described using formal descriptions (mathematical) which specifies the requirements for class members. For example, class Colors would contain all the colors of our interest field: Blue, Green, Orange, Purple, Red, White, Yellow, AllColours.

Classes can be organized in super classes - sub class hierarchy, which is known as taxonomy. Subclasses has specialized the super classes. For example, grades Contents Plants - Plants can be a subclass of Content class (Content is super class and Plants is class). This can be translated as: "All plants are Content", "Plants All class members are members of the class Content". The expression is a relation forms the basic structure of the ontology. 


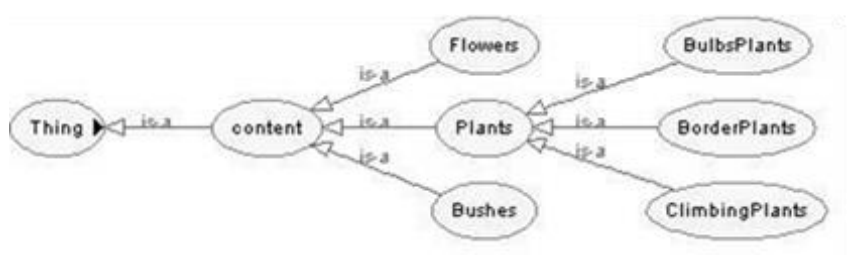

Fig. 1. Relation between classes Content - Plants

If the expression is $A$ is $a B$, it means that node $\mathrm{A}$ is a subtype of node B. For example, BulbsPlants is a Plants class, or Plants is a Content. It should be noted that is a does not mean is an instance of. An instance, ontologically speaking, is a specific example of something; e.g. a BulbsPlants is a Plants, but Gladiola is an instance of a BulbsPlants, rather than a subtype of BulbsPlants.

However, if it's know that BulbsPlants is a Plants, it is similar with every instance of BulbsPlants is a Plants, more over even every instance of BulbsPlants is a content.

Although there is no binding agreement on the appointment of OWL classes, it is recommended that all class names begin with a capital letter and should not contain spaces, for example:

- Garden,

- ThunbergiaAlata,

- BulbsPlants,

- BorderPlants,

- LongBlooming.

Alternatively, it can use the underscore character to group words Hedera_Iedera, Bluebells_Viorele.

Empty ontology contains a class called owl: Thing. OWL classes are interpreted as sets of individuals and are constructed from descriptions of the conditions that must be met by an individual to closely match a member of class. OWL ontology is a set of axioms, which provide explicit logical assertions about three types of things: classes, individuals and properties.

Using reasoner it can infer other facts which are inevitably contained in the ontology, for example if an individual Anemone is in class BulbsPlants, and the class BulbsPlants is a subclass of the class Plants, a reasoner will infer that Anemone is a Plants.

It was used the next types of axioms that can be expressed in OWL 2 and also the Manchester syntax:

- Class declaration defines a class. A class may contain individuals or other subclass.

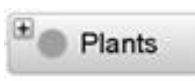

Declaration( Class( :Plants ) )

- Individual declaration defines a named individual.

\section{Anemone}

Declaration( NamedIndividual( :Anemone )

- Class assertion state that an individual belongs to a class:

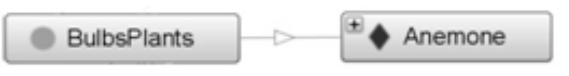

ClassAssertion( :BulbsPlants :Anemone )

- Subclass assertion declares that all individuals that belong to a class belong also to another class:

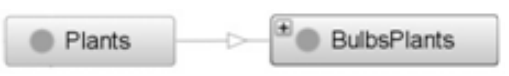

SubClassOf( :BulbsPlants :Plants )

Property declaration defines a data property to link an individual to data, or object property to link to an individual:

Declaration(DataProperty(:SpaceBetweenPlants))

- Property assertion issue the relation of an individual to either data or individual:

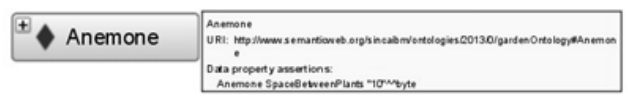

DataPropertyAssertion(:SpaceBetweenPlants :Anemone "10"^^xsd:byte)

Before it will be presented the relationships between classes it must have to list the class hierarchy: here was used the Ontograf images produced by Protégé OWL editor. In the plants environment any scene consists of Blooming, Caring, Colors, Content, Shadows, Toxic. These will be the super classes of the Garden ontology (Fig.2).

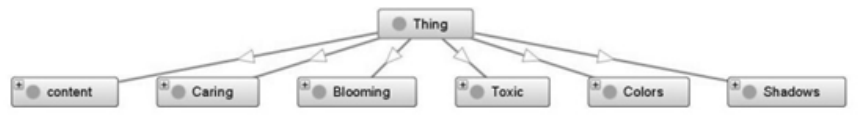

Fig. 2. Garden ontology super classes

A landscape may refer to Blooming, Caring, Colors, Content, Shadows, Toxic environment. The content has been adapted to these situations. Further classes are customized by introducing appropriate subclasses: Blooming is refered by LongBlooming or ShortBlooming, Caring consists of HardCaring or SimpleCaring, Colors prevailing Blue, Green, Orange, Purple, Red, White, Yellow, AllColours, Content defines Bushes, Flowers and Plants where Pants could be BorderPlants, BulbsPlants and ClimbingPlants species, Shadows might be NaturalLight, Semidarkness and Shadow, Toxic as it may be isToxic, notToxic or SlightlyToxic. The figure below shows a representation of the hierarchy classes in Garden ontology (Fig. 3). It is presents an overall graph of Garden ontology, emphasizing the relationship among the main classes of the ontology. The word concept is sometimes used 
instead of classes. Classes are concrete representations of concepts.

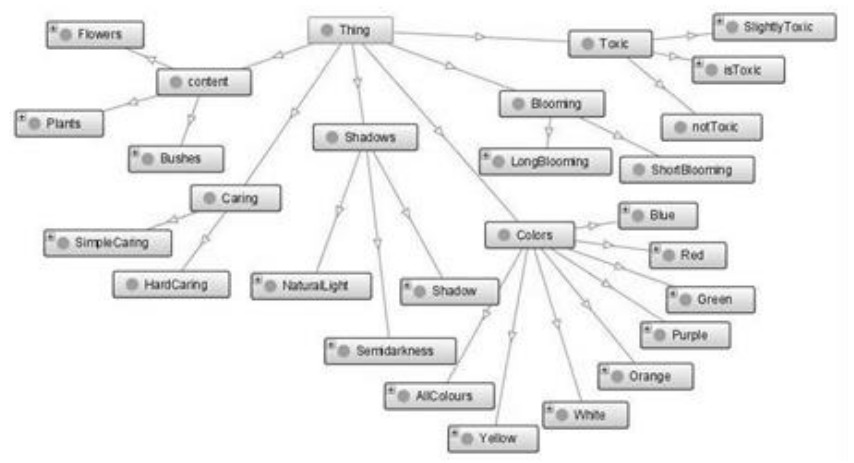

Fig. 3. Class hierarchy

\section{RESULTS AND DISCUSSION}

In this section, it is described the design considerations and modeling concepts, together with a landscape scenario used to illustrate own ideas in that context. The ontology is not complete until the addition of individuals. For every class it can be established as many individuals are necessary. For this ontology it was considered that some individuals for a class are enough. As the individuals are added it was associated the appropriate object and data properties like Figure 4a. In Figure $4 \mathrm{~b}$ there is an example of individual with description and property assertions. In the Figure $4 \mathrm{c}$ was represented all individuals with properties isPerfumed.

For proper experiment it was implemented an ontology with 25 entities (Individuals), 111 relations (Fig. 4a) and 9 data properties. The tests have been carried out using a reasoned and the results have been validated by an expert. On the basis of the description (conditions) a class reasoner can check whether or not that class may have an instance. A class is declared inconsistent if it can have any instance. OWL provides set operations in their usual mathematical meaning.

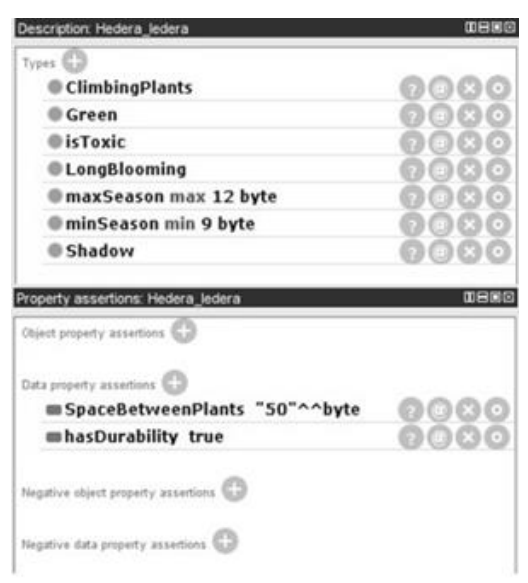

(a)

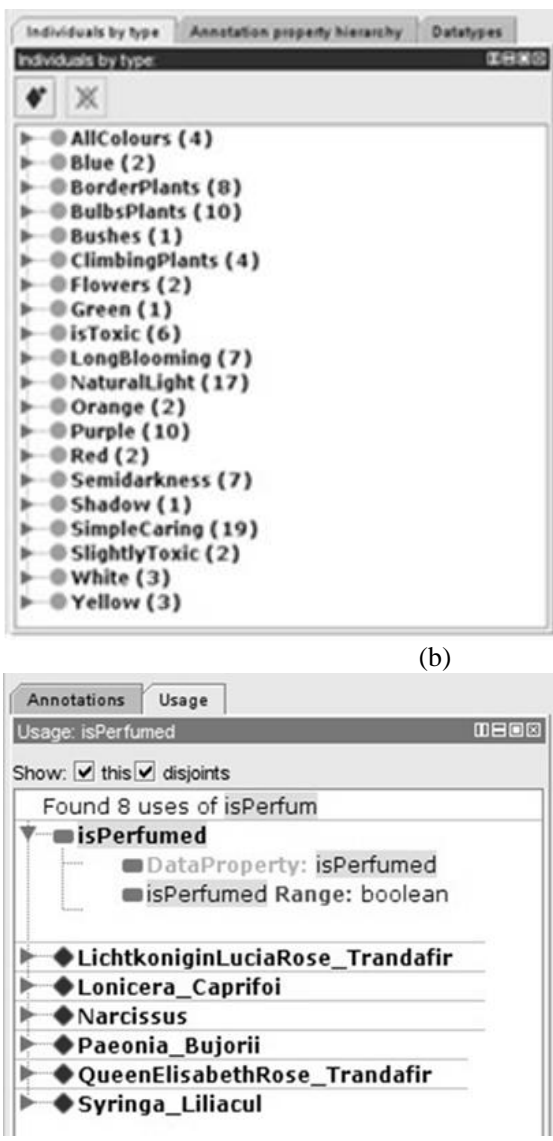

(c)

Fig. 4. (a) Individuals by type; (b) Description and property assertions; (c) Individuals with a property

It is display the set operations on an example in Figure 5. It was defined a class ClimbingPlants which contains four new individuals named SmallBoy, BigBoy, SmallGirl, BigGirl. These individuals must be declared as different from each other, otherwise an OWL reasoner expects that they may be the same:

Declaration(Class(:ClimbingPlants))

Declaration(NamedIndividual(:Hedera_Iedera))

Declaration(NamedIndividual(:Ipomoea_Zorele))

Declaration(NamedIndividual(:Lonicera_Caprifoi)))

Declaration(NamedIndividual(:ThunbergiaAlata))

ClassAssertion(:ClimbingPlants:Hedera_Iedera)

ClassAssertion(:ClimbingPlants:Ipomoea_Zorele)

ClassAssertion(:ClimbingPlants:Lonicera_Caprifoi)

ClassAssertion(:ClimbingPlants:ThunbergiaAlata)

DifferentIndividuals(:Hedera_Iedera

:Ipomoea_Zorele

:Lonicera_Caprifoi) :ThunbergiaAlata)

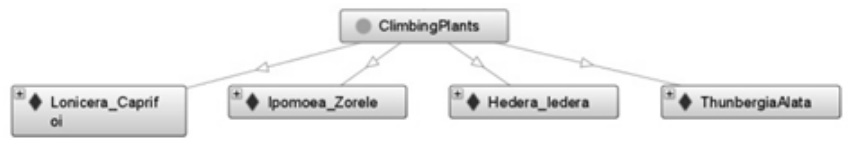

Fig. 5. ClimbingPlants class 
For data properties it was necessary to restrict the values of a data property. For example the axiom:

ClassAssertion(DataMinCardinality(15:minHeight

xsd:byte):Anemone)

ClassAssertion(DataMinCardinality(3:minSeason

xsd:byte):Anemone)

ClassAssertion(DataMaxCardinality(25:maxHeight

xsd:byte):Anemone)

ClassAssertion(DataMaxCardinality(5:maxSeason

xsd:byte):Anemone)

DataPropertyAssertion(:SpaceBetweenPlants:Anemone

"10"^^xsd:byte)

Without having a reasoner is very difficult to maintain large ontology in a state logically correct.

In cases where ontology has classes that have multiple super classes, it is important that every time you build a tree hierarchy of classes that simple. The duty of the reasoner is to calculate and maintain multiple rights (Fig. 6).

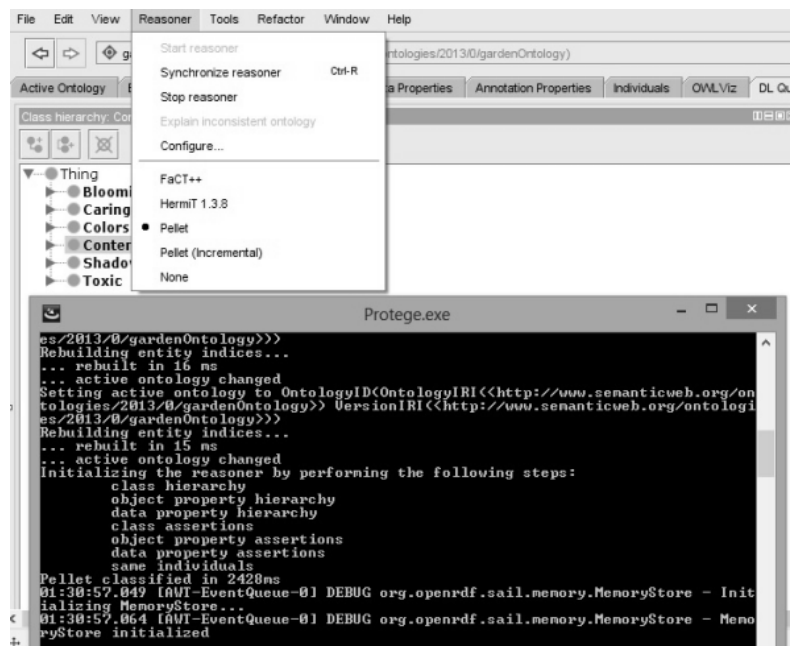

Fig. 6. Garden ontology processed by a reasoner

For validating the ontology, there are testing several sessions of reasoning. The most important constraints ([40]) that can be specified are:

- allValuesFrom - which specifies that all values of the properties are in a certain area;

- someValuesFrom - which specify that a property has values in a particular field of cardinality;

- hasValue - which can be either an individual or a data value. A restriction containing hasValue constraint describes a class of all individuals for which the property concerned has at least one value semantically equal to specificated value (it may have other values as well);

- minCardinality - describes a class of all individuals that have at least $\mathrm{N}$ semantically distinct values (individuals or data values) for the property concerned, where $\mathrm{N}$ is the value of the cardinality constraint;
maxCardinality - describes a class of all individuals that have at most $\mathrm{N}$ semantically distinct values (individuals or data values) for the property concerned, where $\mathrm{N}$ is the value of the cardinality constraint.

In Figure 7 was observed that composing relationships hasDurability and isPerfumed will obtain exactly as result the plants with this properties. It was testing the next query:

\section{(hasDurability value true) and (isPerfumed value true)}

The result was:

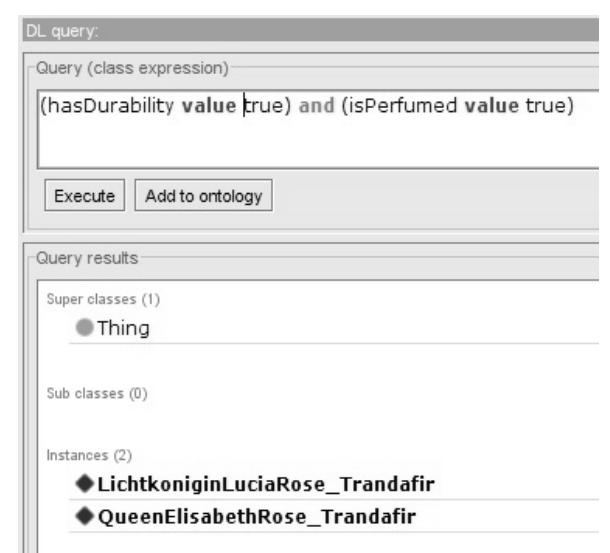

Fig. 7. Perfumed and Durability plants

In order to determine all the bulbs plants which growing after May, the proper query is:

\section{BulbsPlants and minSeason min 5}

It was obtained as result: Gladiola, Ixia, Dahlia, IrisHollandica, MaryLily (Figure 8a). To find bulbs plants which are Toxic and have property to need space between plants minimum $5 \mathrm{~cm}$ the next query was executed:

\section{BulbsPlants and isToxic and SpaceBetweenPlants some byte [ $>=5$ ]}

Result is showed in Figure 8b:

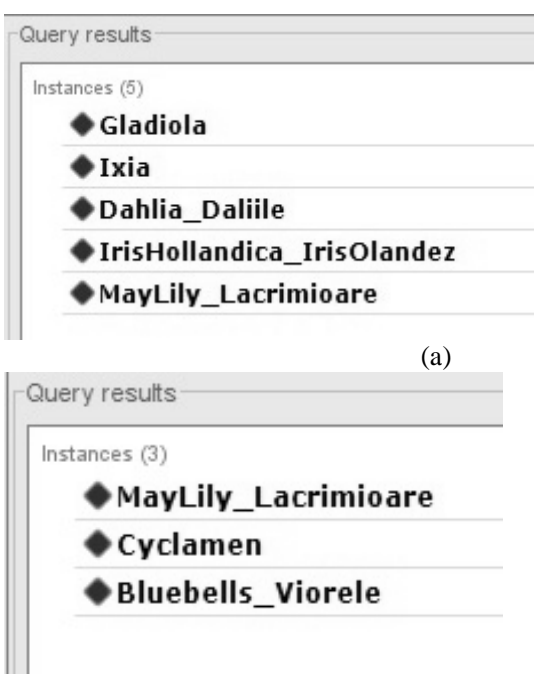

(b) 
Fig. 8. (a) Bulbs plants growing after May; (b) Toxic bulbs plants with specified space between plants

To find out the simple caring plants, need natural light and belong one of the next categories: bushes, flowers or climbing plants it is enough to run the next query:

\section{(Bushes or Flowers or ClimbingPlants) and SimpleCaring and NaturalLight}

The result was:

\begin{tabular}{||} 
Super classes (3) \\
Content \\
NaturalLight \\
SimpleCaring \\
Instances (4) \\
LichtkoniginLuciaRose_Trandafir \\
Ipomoea_Zorele \\
Q SueenElisabethRose_Trandafir
\end{tabular}

Fig. 9. The simple caring plants, natural light, bushes, flowers or climbing plants

\section{CONCLUSIONS AND FUTURE WORK}

When is necessary to define complex ontology, and beyond, Protégé is recommended. With a highly developed visual interface can define classes, individuals, properties, relations only by a few clicks thus saving much time working with writing effective in OWL.

It was developed genetic algorithms applied to the problems of composition of the sustainable landscapes. Future research can be focused on deepen the study of genetic algorithms and adaptive algorithms to find ways of applying evolutionary computation to solve them. It will mainly consider the transposition of the sustainable landscapes. Theoretical solutions will be integrated into a practical application, usable in real life.

The future research would be interesting to demonstrate that the proposed system is capable of operating in complex areas and can handle large data sets, which can compete with similar systems.

\section{REFERENCES}

[1] P. Selman, "What do we mean by "sustainable landscape"?," Sustainability: Science, Practice and Policy 4(2): 23 - 28, 2008.

[2] F.T. Edum-Fotwe and A.D.F. Price, "A social ontology for appraising sustainability of construction projects and developments," International Journal of Project Management 27(4): 313-322, 2009.

[3] D. Ciszewski, P. Bijata and K. Klimek, "Reconstruction of post-mining attenuation of heavy metal pollution in sediment of the zlatýpotok, eastern Sudetymts," Carpathian Journal of Earth and Environmental Sciences, 9(4): 109 - 120, 2014.

[4] D. Petrea, S. Bilaşco, S. Roșca, I. Vescan and I. Fodorean, "The determination of the landslide occurrence probability by gis spatial analysis of the land morphometric characteristics (case study: the Transylvanian plateau)," Carpathian Journal of Earth and Environmental Sciences 9(2): 91 - 102, 2014.
[5] G. Macovei and R. Givulescu, "The present stage in the knowledge of the Fossil flora at Chiuzbaia," Maramureş, Romania. Carpathian Journal of Earth and Environmental Sciences 1(1): 41 - 52, 2006.

[6] S. Hassan, O. Soliman and A. Mahmoud, "Optimized data input for the support vector machine classifier using aster data," Case study: WadiAtalla area, Eastern Desert, Egypt, Carpathian Journal of Earth and Environmental Sciences, 10(1): 15 - 26, 2015.

[7] S. C. Park, "Sustainable Design and Historic Preservation," Guiding Principles for Sustainable Design, NPS Denver Service Center CRM No 2-1998, 1993.

[8] C. R. Jacobson, "Identification and quantification of the hydrological impacts of imperviousness in urban catchments: A review," Journal of Environmental Management 92: 1438 - 1448, 2011.

[9] T. D. Fletcher and P. H. Andrieu, "Understanding, management and modelling of urban hydrology and its consequences for receiving waters; a state of the art review," Advances in Water Resources 51: 261 - 279, 2013.

[10] M. C. Stoecklein, "The Complete Plant Selection Guide for Landscape Design," Purdue University Press, West Lafayette, Indiana, ISBN 155753-139-0, 2001.

[11] M. Calkins, "Strategy use and challenges of ecological design in landscape architecture," Landscape and Urban Planning 73(1): 29 - 48, 2011.

[12] A. Gordon, D. Simondson, M. White, A. Moilanen, and S. A. Bekessy, "Integrating conservation planning and landuseplanning in urban landscapes," Landscape and Urban Planning 91(4): 183 - 194, 2009.

[13] M. Scholz, and S. K. Yazdi, "Treatment of road runoff by a combined storm water treatment, detention and infiltration system," Water Air Soil Pollut. 198 (1-4): 55-64, 2009.

[14] F. Kazemi, S. Beecham, and J. Gibbs, "Streetscale bioretention basins in Melbourne and their effect on local biodiversity," Ecological Engineering, 35(10): 1454 - 1465, 2009.

[15] F. Kazemi, S. Beecham, and J. Gibbs, "Streetscape biodiversity and the role of bioretention swales in an Australian urban environment," Landscape and Urban Planning, 101(2): 139 - 148, 2011.

[16] S. A. Trowsdale, and R. Simcock, "Urban stormwater treatment using bioretention," Journal of Hydrology, 397 (3-4), 3:167 - 174, 2011.

[17] D. A. Levinthal, "Landscape Design: Designing for Local Action in Complex Worlds," Organization Science: 342 - 357, 1999.

[18] J. L. Motloch, "Introduction to Landscape Design," ISBN 0-471-352918, Printed in the U.S.A, 2000.

[19] J. Makhzoumi, and G. Pungetti, "Ecological landscape design and planning," Taylor and Francis e-Library, 2005.

[20] N. Dunnett, and J. Hitchmough, "The dynamic landscape," British Library Cataloguing, Publication Data, ISBN 0-203-40287-1 Master ebook ISBN, 2004.

[21] M. Mahdavinejada, and M. Abedi, "Community -oriented landscape design for sustainability in architecture and planning," Procedia Engineering 21: 337 - 344, International Conference on Green Buildings and Sustainable Cities, 2011.

[22] T. Bata, G. Mezösi, and B. Meyer, "Landscape units for hungary using multiresolution segmentation of geo-data and fuzzy analysis," Carpathian Journal of Earth and Environmental Sciences, 9(2): 45 - 56, 2014.

[23] J. O. Simonds, and B. Star, "Landscape Architecture," Fourth Edition: A Manual of Land Planning and Design, McGraw Hill book Company (Inc.), New York 6, 2006.

[24] C. W. Huang, Y. P. Lin, T. S. Ding, and J. Anthony, "Developing a Cell-Based Spatial Optimization Model for Land - Use Patterns Planning," Sustainability 6, 9139-9158, 2014.

[25] R. M. de Waal, and S. Stremke, "Energy Transition: Missed Opportunities and Emerging Challenges for Landscape Planning and Designing," Sustainability 6, 4386-4415, 2014.

[26] S. Kraines, and W. Guo, "A System for Ontology-Based Sharing of Expert Knowledge in Sustainability Science," Data Science Journal 9: $107-123,2010$ 
[27] E. Bakay, "The role of housing estates' green surfaces in forming the city climate of Budapest," Applied Ecology and Environmental Research 10(1): 1 - 16, 2012.

[28] D. Takács, D. K. Varró, and E. Bakay, "Comparison of different space indexing methods for ecological evaluation of urban open spaces," Applied Ecology and Environmental Research 12(4): 1027 - 1048, 2014.

[29] E. Z. Başkent, "Combinatorial Optimization in Forest Ecosystem Management Modeling," Turkish Journal of Agriculture and Forestry 25(3): 187-194, 2001.

[30] J. Biolchini, "Developing a UMLS-based Ontology of Cardiology Procedures for Cognitive Support in Medical Decision Making," Medical Informatics Training Program, National Library of Medicine, 2002.

[31] G. Giray, and M. U. Ünalir, "A method for ontology-based semantic relatedness measurement," Turkish Journal of Electrical Engineering and Computer Sciences 21: 420 - 438, 2013.

[32] N. F. Noy, and D. L. McGuinness, “Ontology Development 101: A Guide to Creating Your First Ontology," Stanford University, Stanford, CA, 94305, 2001.

[33] A. Cernian, D. Carstoiu, O. Vasilescu, and A. Olteanu, "OntoLaw Ontology Based Legal Management and Information Retrieval System," Journal of Control Engineering and Applied Informatics, Vol.15, No.4 pp. $77-85,2013$.
[34] O. Matei, D. Contras, and P. C. Pop, "Applying evolutionary computation for evolutionary ontologies," in Proc. of IEEE Congress on Evolutionary Computation (CEC-2014): 1520 - 1527, Beijing, China, 611 July, 2014.

[35] I. Horrocks, and S. Ulrike, "Ontology reasoning in the SHOQ (D) description logic," International Joint Conference on Artificial Intelligence 1(3), 2001.

[36] M. Fernandez-Lopez, and A. Gomez-Perez, "Overview and analysis of methodologies for building ontologies," Knowledge Engineering Review, 17(2): 129-156, 2002.

[37] E. Biesalski, and A. Abecker, "Human Resource Management with Ontologies," 3rd Conference Professional Knowledge Management, Experience and Visions, Springer, LNCS vol. 3728, 2005.

[38] M. Laukkanen, and H. Helin, "Competence Manager - Applying the Semantic Web in Practice," 3rd International Semantic Web Conference (ISWC 2004).

[39] S. Furst, R. Blackburn, and B. Rosen, "Virtual team effectiveness: a proposed research agenda," Information Systems Journal 9(4): 249 269, 1999.

[40] S. Bechhofer, F. van Harmelen, J. Hendler, I. Horrocks, D. L. McGuinness, P. F. Patel-Schneider, L. A. Stein, and F. W. Olin, "OWL Web Ontology Language Reference," [Online]. Available: http://www.w3.org/TR/owl-ref/\#allValuesFrom-def, 2004. 